\title{
Entropic Corrections to Friedmann Equations
}

\author{
Ahmad Sheykhi * \\ Department of Physics, Shahid Bahonar University, P.O. Box 76175, Kerman, Iran \\ Research Institute for Astronomy and Astrophysics of Maragha (RIAAM), Maragha, Iran
}

\begin{abstract}
Recently, Verlinde discussed that gravity can be understood as an entropic force caused by changes in the information associated with the positions of material bodies. In the Verlinde's argument, the area law of the black hole entropy plays a crucial role. However, the entropy-area relation can be modified from the inclusion of quantum effects, motivated from the loop quantum gravity. In this note, by employing this modified entropy-area relation, we derive corrections to Newton's law of gravitation as well as modified Friedman equations by adopting the viewpoint that gravity can be emerged as an entropic force. Our study further supports the universality of the log correction and provides a strong consistency check on Verlinde's model.

PACS: 04.20.Cv, 04.50.-h, 04.70.Dy.
\end{abstract}

It was first pointed out by Jacobson [1] that the hyperbolic second order partial differential Einstein equation has a predisposition to the first law of thermodynamics. This profound connection between the first law of thermodynamics and the gravitational field equations has been extensively observed in various gravity theories [2, 3]. Recently the study on the connection between thermodynamics and gravity has been generalized to the cosmological situations [4 9], where it was shown that the differential form of the Friedmann equation on the apparent horizon in the FRW universe can be rewritten in the form of the first law of thermodynamics. The extension of this connection has also been carried out in the braneworld cosmology [10, 11]. The deep connection between the gravitational equation describing the gravity in the bulk and the first law of thermodynamics on the apparent horizon reflects some deep ideas of holography. Although Jacobson's derivation is logically clear and theoretically sound, the statistical mechanical origin of the thermodynamic nature of general relativity remains obscure.

Recently, Verlinde [12 has put forward an idea similar in spirit to Jacobson's thermodynamic derivation of the Einstein equations, where it is argued that Newton's law of gravitation can be understood as an entropic force caused by information changes when a material body moves away from the holographic screen. Quantitatively, when a test particle or excitation moves apart from the holographic screen, the magnitude of the entropic force on this body has the form

$$
F \triangle x=T \triangle S,
$$

where $\triangle x$ is the displacement of the particle from the holographic screen, while $T$ and $\triangle S$ are the temperature and the entropy change on the screen, respectively. Verlinde's derivation of Newton's law of gravitation at the very least offers a strong analogy with a well understood statistical mechanism. Therefore, this derivation opens a

\footnotetext{
*sheykhi@mail.uk.ac.ir
}

new window to understand gravity from the first principles. The study on the entropic force has raised a lot of enthusiasm recently (see [13 16] and references therein).

It is important to note that in Verlinde discussion, the black hole entropy $S$ plays a crucial role in the derivation of Newton's law of gravitation. Indeed, the derivation of Newton's law of gravity depends on the entropy-area relationship $S=A / 4 \ell_{p}^{2}$ of black holes in Einsteins gravity, where $A=4 \pi R^{2}$ represents the area of the horizon and $\ell_{p}^{2}=G \hbar / c^{3}$ is the Planck length. However, this definition can be modified from the inclusion of quantum effects, motivated from the loop quantum gravity (LQG). The quantum corrections provided to the entropy-area relationship leads to the curvature correction in the EinsteinHilbert action and vice versa [17, 18]. The corrected entropy takes the form [19]

$$
S=\frac{A}{4 \ell_{p}^{2}}-\beta \ln \frac{A}{4 \ell_{p}^{2}}+\gamma \frac{\ell_{p}^{2}}{A}+\text { const }
$$

where $\beta$ and $\gamma$ are dimensionless constants of order unity. The exact values of these constants are not yet determined and still an open issue in loop quantum cosmology. These corrections arise in the black hole entropy in LQG due to thermal equilibrium fluctuations and quantum fluctuations [20]. Taking the corrected entropy-area relation (2) into account, we will derive the corrections to the Newton's law of gravitation as well as the modified Friedman equations. We rewrite Eq. (2) in the following form

$$
S=\frac{A}{4 \ell_{p}^{2}}+s(A)
$$

where $s(A)$ stands for the correction terms in the entropy expression. We adopt the viewpoint of [12]. Suppose we have two masses one a test mass and the other considered as the source with respective masses $m$ and $M$. Centered around the source mass $M$, is a spherically symmetric surface $\mathcal{S}$ which will be defined with certain properties that will be made explicit later. To derive the entropic law, the surface $\mathcal{S}$ is between the test mass and the source mass, but the test mass is assumed to be very close to the 
surface as compared to its reduced Compton wavelength $\lambda_{m}=\frac{\hbar}{m c}$. When a test mass $m$ is a distance $\Delta x=$ $\eta \lambda_{m}$ away from the surface $\mathcal{S}$, the entropy of the surface changes by one fundamental unit $\triangle S$ fixed by the discrete spectrum of the area of the surface via the relation

$$
\triangle S=\frac{\partial S}{\partial A} \triangle A=\left(\frac{1}{4 \ell_{p}^{2}}+\frac{\partial s(A)}{\partial A}\right) \triangle A .
$$

The energy of the surface $\mathcal{S}$ is identified with the relativistic rest mass of the source mass:

$$
E=M c^{2} .
$$

On the surface $\mathcal{S}$, there live a set of "bytes" of information that scale proportional to the area of the surface so that

$$
A=Q N,
$$

where $N$ represents the number of bytes and $Q$ is a fundamental constant which should be specified later. Assuming the temperature on the surface is $T$, and then according to the equipartition law of energy 21], the total energy on the surface is

$$
E=\frac{1}{2} N k_{B} T .
$$

Finally, we assume that the force on the particle follows from the generic form of the entropic force governed by the thermodynamic equation of state

$$
F=T \frac{\triangle S}{\triangle x},
$$

where $\triangle S$ is one fundamental unit of entropy when $|\triangle x|=\eta \lambda_{m}$, and the entropy gradient points radially from the outside of the surface to inside. Note that $N$ is the number of bytes and thus $\triangle N=1$, hence from (6) we have $\triangle A=Q$. Now, we are in a position to derive the entropic-corrected Newton's law of gravity. Combining Eqs. (4)- (8), we easily obtain

$$
F=-\frac{M m}{R^{2}}\left(\frac{Q c^{3}}{2 \pi k_{B} \hbar \eta}\right)\left[\frac{1}{4 \ell_{p}^{2}}+\frac{\partial s}{\partial A}\right]_{A=4 \pi R^{2}},
$$

This is nothing but the Newton's law of gravitation to the first order provided we define $Q=8 \pi k_{B} \eta \ell_{p}^{4}$. Thus we reach

$$
F=-\frac{G M m}{R^{2}}\left[1+4 \ell_{p}^{2} \frac{\partial s}{\partial A}\right]_{A=4 \pi R^{2}},
$$

Finally, using Eq. (2) we obtain the modified Newton's law of gravitation as

$$
F=-\frac{G M m}{R^{2}}\left[1-\frac{\beta}{\pi} \frac{\ell_{p}^{2}}{R^{2}}-\frac{\gamma}{4 \pi^{2}} \frac{\ell_{p}^{4}}{R^{4}}\right],
$$

Thus, with the correction in the entropy expression, we see that the Newton's law will modified accordingly. As we mentioned, these corrections are well motivated from bottom-up quantum gravity theories. The log correction to the area-entropy relation appears to have an almost universal status, having been derived from multiple different approaches to the calculation of entropy from counting microscopic states in different quantum gravity models. Since the last two terms in Eq. (11) can be comparable to the first term only when $R$ is very small, the corrections make sense only at the very small distances. When $R$ becomes large, the entropy-corrected Newton's law reduces to the usual Newton's law of gravitation.

Let us compare our result with that obtained in [15]. The first correction term originates from the log correction in Eq. (17) of [15] is similar to ones we obtained in Eq. (11), however, it seems that the second correction term in Eq. (17) of [15] is not reasonable. Physically, the effect of the correction terms on the quantity should be less than the uncorrected quantity. Similarly, the contribution of the first correction term in the physical quantity (force here) should be more from the second term and so on. For all above reasons, we think the second correction term in Eq. (17) of 15 is not correct, and the corrected form is that presented in our note. The origin of this difference is due to the fact that the second (volume) correction term to the entropy expression in [15] is not indeed a correction term, although it was argued by [15] that this term is also emerged in a model for the microscopic degrees comprising the black hole entropy in LQG [23]. Let us stress here that although in the literature there is doubt about the second correction term in entropy-corrected relation, however, it is widely believed [19] that the next quantum correction term to black hole entropy have the form $\ell_{p}^{2} / A$, which leads to the resonable correction terms to Newton's law of gravitation (11) as we have shown in the present work and will lead to corrected modified Friedmann equation as we will see later.

Next, we extend our discussion to the cosmological setup. Assuming the background spacetime to be spatially homogeneous and isotropic which is given by the Friedmann-Robertson-Walker (FRW) metric

$$
d s^{2}=h_{\mu \nu} d x^{\mu} d x^{\nu}+R^{2}\left(d \theta^{2}+\sin ^{2} \theta d \phi^{2}\right),
$$

where $R=a(t) r, x^{0}=t, x^{1}=r$, the two dimensional metric $h_{\mu \nu}=\operatorname{diag}\left(-1, a^{2} /\left(1-k r^{2}\right)\right)$. Here $k$ denotes the curvature of space with $k=0,1,-1$ corresponding to open, flat, and closed universes, respectively. The dynamical apparent horizon, a marginally trapped surface with vanishing expansion, is determined by the relation $h^{\mu \nu} \partial_{\mu} R \partial_{\nu} R=0$. A simple calculation gives the apparent horizon radius for the FRW universe

$$
R=a r=\frac{1}{\sqrt{H^{2}+k / a^{2}}} .
$$

We also assume the matter source in the FRW universe is a perfect fluid with stress-energy tensor

$$
T_{\mu \nu}=(\rho+p) u_{\mu} u_{\nu}+p g_{\mu \nu} .
$$


Due to the pressure, the total mass $M=\rho V$ in the region enclosed by the boundary $\mathcal{S}$ is no longer conserved, the change in the total mass is equal to the work made by the pressure $d M=-p d V$, which leads to the well-known continuity equation

$$
\dot{\rho}+3 H(\rho+p)=0
$$

where $H=\dot{a} / a$ is the Hubble parameter. It is instructive to first derive the dynamical equation for Newtonian cosmology. Consider a compact spatial region $V$ with a compact boundary $\mathcal{S}$, which is a sphere with physical radius $R=a(t) r$. Note that here $r$ is a dimensionless quantity which remains constant for any cosmological object partaking in free cosmic expansion. Combining the second law of Newton for the test particle $m$ near the surface, with gravitational force (11) we get

$$
F=m \ddot{R}=m \ddot{a} r=-\frac{G M m}{R^{2}}\left[1-\frac{\beta}{\pi} \frac{\ell_{p}^{2}}{R^{2}}-\frac{\gamma}{4 \pi^{2}} \frac{\ell_{p}^{4}}{R^{4}}\right],
$$

We also assume $\rho=M / V$ is the energy density of the matter inside the the volume $V=\frac{4}{3} \pi a^{3} r^{3}$. Thus, Eq. (16) can be rewritten as

$$
\frac{\ddot{a}}{a}=-\frac{4 \pi G}{3} \rho\left[1-\frac{\beta}{\pi} \frac{\ell_{p}^{2}}{R^{2}}-\frac{\gamma}{4 \pi^{2}} \frac{\ell_{p}^{4}}{R^{4}}\right],
$$

This is nothing but the entropy-corrected dynamical equation for Newtonian cosmology. The main difference between this equation and the standard dynamical equation for Newtonian cosmology is that the correction terms now depends explicitly on the radius $R$. However, we can remove this confusion. Assuming that for Newtonian cosmology the spacetime is Minkowskian with $k=0$, then we get $R=1 / H$, and we can rewrite Eq. (17) in the form

$$
\frac{\ddot{a}}{a}=-\frac{4 \pi G}{3} \rho\left[1-\frac{\beta \ell_{p}^{2}}{\pi}\left(\frac{\dot{a}}{a}\right)^{2}-\frac{\gamma \ell_{p}^{4}}{4 \pi^{4}}\left(\frac{\dot{a}}{a}\right)^{4}\right] .
$$

It was argued in [13] that for deriving the Friedmann equations of FRW universe in general relativity, the quantity that produces the acceleration is the active gravitational mass $\mathcal{M}$ [22], rather than the total mass $M$ in the spatial region $V$. With the entropic corrections terms, the active gravitational mass $\mathcal{M}$ will also modified as well. On one side, from Eq. (17) with replacing $M$ with $\mathcal{M}$ we have

$$
\mathcal{M}=-\frac{\ddot{a} a^{2}}{G} r^{3}\left[1-\frac{\beta}{\pi} \frac{\ell_{p}^{2}}{R^{2}}-\frac{\gamma}{4 \pi^{2}} \frac{\ell_{p}^{4}}{R^{4}}\right]^{-1} .
$$

On the other side, the active gravitational mass is defined as 13.

$$
\mathcal{M}=2 \int_{V} d V\left(T_{\mu \nu}-\frac{1}{2} T g_{\mu \nu}\right) u^{\mu} u^{\nu}
$$

A simple calculation leads

$$
\mathcal{M}=(\rho+3 p) \frac{4 \pi}{3} a^{3} r^{3} .
$$

Equating Eqs. (19) and (21) we find

$$
\frac{\ddot{a}}{a}=-\frac{4 \pi G}{3}(\rho+3 p)\left[1-\frac{\beta}{\pi} \frac{\ell_{p}^{2}}{R^{2}}-\frac{\gamma}{4 \pi^{2}} \frac{\ell_{p}^{4}}{R^{4}}\right] .
$$

This is the modified acceleration equation for the dynamical evolution of the FRW universe. Multiplying $\dot{a} a$ on both sides of Eq. (22), and using the continuity equation (15), after integrating we find

$H^{2}+\frac{k}{a^{2}}=\frac{8 \pi G}{3} \rho\left[1-\frac{\beta}{\pi} \frac{\ell_{p}^{2}}{\rho R^{2}} \int \frac{d\left(\rho a^{2}\right)}{a^{2}}-\frac{\gamma}{4 \pi^{2}} \frac{a^{2} \ell_{p}^{4}}{\rho R^{4}} \int \frac{d\left(\rho a^{2}\right)}{a^{4}}\right]$.

Now, in order to calculate the integrations in the correction terms we need to find $\rho=\rho(a)$. Assume the equation of state parameter $w=p / \rho$ is a constant, the continuity equation (15) can be integrated immediately to give

$$
\rho=\rho_{0} a^{-3(1+w)},
$$

where $\rho_{0}$, an integration constant, is the present value of the energy density. Inserting relation (24) in Eq. (23), after integration, we obtain

$H^{2}+\frac{k}{a^{2}}=\frac{8 \pi G}{3} \rho\left[1-\frac{\beta(1+3 w)}{3 \pi(1+w)} \frac{\ell_{p}^{2}}{R^{2}}-\frac{\gamma(1+3 w)}{4 \pi^{2}(5+3 w)} \frac{\ell_{p}^{4}}{R^{4}}\right]$.

Using Eq. (13) we can further rewrite the above equation as

$$
\begin{aligned}
& \left(H^{2}+\frac{k}{a^{2}}\right)\left[1-\frac{\beta \ell_{p}^{2}(1+3 w)}{3 \pi(1+w)}\left(H^{2}+\frac{k}{a^{2}}\right)\right. \\
& \left.-\frac{\gamma \ell_{p}^{4}(1+3 w)}{4 \pi^{2}(5+3 w)}\left(H^{2}+\frac{k}{a^{2}}\right)^{2}\right]^{-1}=\frac{8 \pi G}{3} \rho .
\end{aligned}
$$

If $\beta$ and $\gamma$ are viewed as small quantities, then the above equation can be expanded up to the linear order of $\beta$ and $\gamma$. The result is

$$
\begin{aligned}
& \left(H^{2}+\frac{k}{a^{2}}\right)+\frac{\beta \ell_{p}^{2}(1+3 w)}{3 \pi(1+w)}\left(H^{2}+\frac{k}{a^{2}}\right)^{2} \\
& +\frac{\gamma \ell_{p}^{4}(1+3 w)}{4 \pi^{2}(5+3 w)}\left(H^{2}+\frac{k}{a^{2}}\right)^{3}=\frac{8 \pi G}{3} \rho,
\end{aligned}
$$

which is in complete agreement with the result of [8] (see also [9]). In this way we derive the entropy-corrected Friedmann equation of FRW universe by considering gravity as an entropic force caused by changes in the information associated with the positions of material bodies. In the absence of the correction terms $(\beta=0=\gamma)$, one recovers the well-known Friedmann equation in standard cosmology. Since the last two terms in Eq. (25) can 
be comparable to the first term only when $a$ is very small, the corrections make sense only at early stage of the universe where $a \rightarrow 0$. When the universe becomes large, the entropy-corrected Friedmann equation reduces to the standard Friedman equation.

In summary, we have shown that with the entropy corrections to the area-relation of the black hole entropy, the Newton's law of gravitation and the Friedmann equations will be modified accordingly. These corrections are motivated from the LQG which is one of the promising theories of quantum gravity. We derived the correction terms to the Newton's law of gravity as well as modified Friedmann equations of the FRW universe starting from the holographic principle and the equipartition law of energy by using Verlinde's argument that gravity appears as an entropic force. In particular, we have found that an apparently universal log correction to the area-entropy, yields deviations from Newton's law and Friedman equations that are identical in form to those obtained from perturbative quantum gravity. This at once sheds light on the reason for the universality of the log correction and provides a strong consistency check on Verlinde's model.

\section{Acknowledgments}

I am grateful to Prof. B. Wang and Prof. R.G. Cai for helpful discussions and reading the manuscript. This work has been supported by Research Institute for Astronomy and Astrophysics of Maragha.
[1] T. Jacobson, Phys. Rev. Lett. 75, 1260 (1995).

[2] C. Eling, R. Guedens, and T. Jacobson, Phys. Rev. Lett. 96, 121301 (2006).

[3] T. Padmanabhan, Phys. Rept. 406 (2005) 49; T. Padmanabhan, Rept. Prog. Phys. 73 (2010) 046901.

[4] M. Akbar and R. G. Cai, Phys. Rev. D 75, 084003 (2007).

[5] R. G. Cai and L. M. Cao, Phys.Rev. D 75, 064008 (2007).

[6] R. G. Cai and S. P. Kim, JHEP 0502, 050 (2005).

[7] B. Wang, E. Abdalla and R. K. Su, Phys.Lett. B 503, 394 (2001);

B. Wang, E. Abdalla and R. K. Su, Mod. Phys. Lett. A 17, 23 (2002);

R. G. Cai and Y. S. Myung, Phys. Rev. D 67, 124021 (2003);

R. G. Cai and L. M. Cao, Nucl. Phys. B 785 (2007) 135.

[8] R. G. Cai, L. M. Cao and Y. P. Hu, JHEP 0808 (2008) 090 .

[9] S. Nojiri and S. D. Odintsov, hep-th/0506212

S. Nojiri and S. D. Odintsov, hep-th70506212;

A. Sheykhi, in preparation.

[10] A. Sheykhi, B. Wang and R. G. Cai, Nucl. Phys. B 779 (2007)1.

[11] A. Sheykhi, B. Wang and R. G. Cai, Phys. Rev. D 76 (2007) 023515;

A. Sheykhi, B. Wang, Phys. Lett. B 678 (2009) 434;

A. Sheykhi, Class. Quantum Grav. 27 (2010) 025007.

[12] E. P. Verlinde, arXiv:1001.0785 [hep-th].

[13] R.G. Cai, L. M. Cao and N. Ohta, Phys. Rev. D 81, 061501(R) (2010).

[14] R.G. Cai, L. M. Cao and N. Ohta, arXiv:1002.113; Yi Ling, Jian-Pin Wu, arXiv:1001.5324

Yun Soo Myung, arXiv:1002.0871;

R. Banerjee, B. R. Majhi, arXiv:1003.2312

S.W. Wei, Y. X. Liu, Y. Q. Wang, arXiv:1001.5238
Y. X. Liu, Y. Q. Wang, S. W. Wei, arXiv:1002.1062

R.A. Konoplya, e-Print: arXiv:1002.2818,

[15] L. Modesto, A. Randono, arXiv:1003.1998.

[16] Y.F. Cai, J. Liu, H. Li, arXiv:1003.4526

[17] T. Zhu and J.R. Ren, Eur. Phys. J. C 62 (2009) 413; R.G. Cai et al, Class. Quantum Grav 26 (2009) 155018; D. A. Easson, arXiv: 1003.1528.

[18] M. R. Banerjee, S. K. Modak, JHEP 0905 (2009) 063; S. K. Modak, Phys. Lett. B 671 (2009) 167; R. Banerjee, S. Gangopadhyay, S. K. Modak, Phys. Lett. B 686 (2010) 181.

[19] J. Zhang, Phys. Lett. B 668 (2008) 353;

R. Banerjee and B. R. Majhi, Phys. Lett. B 662, 62 (2008);

R. Banerjee and B. R. Majhi, JHEP 0806 (2008) 095;

R. Banerjee, B. R. Majhi and S. Samanta, Phys. Rev. D 77, 124035 (2008);

R. Banerjee, B. R. Majhi, Phys. Lett. B 674 (2009) 218; B. R. Majhi, Phys. Rev. D 79 (2009) 044005;

S. Nojiri and S. D. Odintsov, Int. J. Mod. Phys. A 16 (2001) 3273;

J. Lidsey et. al., Phys. Lett. B 544 (2002) 337;

[20] C. Rovelli, Phys. Rev. Lett. 77 (1996) 3288;

A. Ashtekar, J. Baez, A. Corichi, and K. Krasnov, Phys. Rev. Lett. 80 (1998) 904.

[21] T. Padmanabhan, Class. Quantum Grav. 21, 4485 (2004);

T. Padmanabhan, arXiv:0912.3165:

T. Padmanabhan, arXiv:1003.5665

[22] T. Padmanabhan, arXiv:1001.3380 [gr-qc].

[23] L. Smolin, arXiv:1001.3668. 